dade, avec ses rues rectilignes et ses parcs magnifiques, témoigne d'une certaine grandeur dans la conception. Porto est le siège d'une Université et d'un épiscopat. Elle est cependant avant tout une ville commerciale et industrielle. La principale activité économique réside dans le commerce du vin de Porto. Provenant de la vallée du Douro, ce vin est transporté à bord de barques à voiles (Barcos Rabelos) jusqu'aux caves de Vila Nova de Gaia. Il subit là un traitement des plus soignés avant d'être entreposé pour la vente.

\title{
DER GANGES ALS WASSERWEG
}

\author{
FRIEDRICH STANG
}

Die wirtschaftliche Bedeutung der indischen Flüsse liegt in erster Linie in ihrem Beitrag zur Bewässerung und Energiegewinnung, nicht aber in ihrem Wert als Verkehrswege. Die monsunalen Niederschläge bedingen eine äußerst unausgeglichene Wasserführung. Der gewaltige Abfluß während der Regenzeit, der mit sehr großem Materialtransport verbunden ist, verursachte eine zu starke Strömung und häufige Verlegung von Flußarmen und Talwegen sowie Beschädigungen der Uferböschungen. Während der Trockenzeit verteilt sich dagegen der geringe Abfluß über ein viel zu breites Bett, und die Wassertiefe ist nur sehr gering.

So gibt es nur auf dem ganzjährig wasserreichen Brahmaputra, der mit geringem Gefälle eine Landschaft durchfließt, wo zahlreiche Wasserarme und häufige Überschwemmungen den Bau von Landverkehrswegen sehr erschweren, heute noch eine Großschiffahrt. Die kurzen schiffbaren Strecken anderer Flüsse, besonders in den Deltagebieten der Halbinsel, sind ohne große Bedeutung. Das gilt inzwischen auch für die Kanäle, von denen nur wenige ausschließlich für die Schiffahrt gebaut wurden, wie der Orissa-Küsten-Kanal (auf dem seit 1928 der Verkehr teilweise eingestellt ist) und der Buckingham-Kanal im Staate Madras. Die anderen sollten der Bewässerungswirtschaft und ider Schiffahrt dienen, was sich schwer miteinander vereinbaren läßt und wobei die letztere meist zu kurz kommt. Das zeigt sich erst jetzt wieder am Beispiel des oft mit dem Tennessee verglichenen Damodars, bei dem eine Schiffahrt auf dem freien Fluß wegen der unausgeglichenen Wasserführung selbst nach umfangreichen Regulierungsarbeiten nur während einer kurzen Zeit des Jahres möglich gewesen wäre. Hier hat die Damodar Valley Corporation einen Kanal für Bewässerungs- und Schiffahrtszwecke zwischen Durgapur und Tribeni angelegt. Die Bewässerungswirtschaft verlangte aber im ebenen Gelände einen Aufstau in kurzen Abständen, so daß die 22 Schleusen auf $137 \mathrm{~km}$ Kanallänge die Schifffahrt behindern. Die Entnahme von Wasser für Bewässerungszwecke gerade zur Zeit geringer Wasserführung ist den Interessen der Schiffahrt entgegengesetzt. So kommt es, daß mehrere Jahre nach Fertigstellung des Kanals die Schiffahrt noch nicht aufgenommen wurde.

Das monsunale Abflußregime erschwert auch die Schiffahrt auf dem Ganges. Es wird jedoch dadurch etwas abgeschwächt, daß der Ganges und seine großen Nebenflüsse, die von Norden kommen, einen Teil ihres Einzugsgebietes im nivalen Bereich haben. In der heißesten Trockenperiode, den Monaten vor Ausbruch des Monsuns, steht daher eine Abflußspende des Hochgebirges aus der Schneeschmelze zur Verfügung; sie kann aber nur einen Teil der jetzt besonders hohen Verdunstung kompensieren. Während bei den großen Zuflüssen Gandak und Gogra wegen ihres kürzeren Laufes der Ausgleich durch die Schmelzwasser in der Trockenzeit noch deutlich bemerkbar ist, nimmt beim Ganges in dieser Periode selbst nach ihrer Einmündung die Wasserführung weiter ab. In den folgenden Sommermonaten verstärkt dagegen die Schneeschmelze im Hochgebirge noch den von den monsunalen Niederschlägen bedingten Abfluß und bringt große Hochwasser.

Diese ungünstigen Verhältnisse haben dazu geführt, daß der heutige Flußverkehr nur noch mit country boats, kleinen, aus Holz gebauten Schiffen, betrieben wird, die eine Ladefähigkeit von einigen Tonnen haben. Die Fortbewegung geschieht mit Segel 
oder Treideln durch Menschenkraft. Dazu steht eine Besatzung von vier bis fünf Mann, Manjies genannt, zur Verfügung. Die country boats befördern fast ausschließlich landwirtschaftliche Güter. Die Großschiffahrt auf dem Ganges und seinen Nebenflüssen ist seit kurzer Zeit eingestellt worden, nachdem sie über hundert Jahre lang eine wichtige Rolle für den Verkehr - besonders in den nördlich des Ganges gelegenen Landesteilen - gespielt hatte.

Bereits 1834 war auf dem Ganges ein regelmäßiger Dampferdienst eingerichtet worden, der schnell an Umfang gewann und so lukrativ gewesen zu sein scheint, daß schon bald mehrere Gesellschaften auftraten, die sich 1889 zu den Joint Steamer Services zusammenschlossen. Damals ging der Verkehr bis Garhmukteswar am Ganges (etwa auf der Breite von Delhi gelegen), Faizabad am Gogra (= Ghagra), Bagaha am Gandak und Agra am Yamuna flußaufwärts. Die Dampfkraft, die der Schiffahrt anfänglich einen solchen Auftrieb gegeben hatte, trug jedoch nach wenigen Jahrzehnten zu ihrem Niedergang bei. In den fünfziger Jahren des 19. Jahrhunderts begann in Indien der Bau der Eisenbahnen, dem die britische Regierung größtes Interesse zuwandte. Durch Förderung und zahlreiche Konzessionen wuchs der Schienenweg schnell zu einem Konkurrenten heran, dem die wenig organisierte Binnenschiffahrt kaum gewachsen war. Ein weiterer Nachteil war der gleichzeitige Ausbau der Bewässerungswirtschaft, die den Flüssen erhebliche Wassermengen entnahm. Sie führte auf dem Ganges zu einer Verminderung der Fahrwassertiefe und einer Aufgabe der Schiffahrt oberhalb Allahabad. Später wurden Buxar am Ganges und Barhaj am Gogra die Endpunkte des Verkehrs und Patna wichtigster Hafenplatz. Vor allem unterhalb Patna, nach Einmündung von Gogra und Gandak, konnten sich die Dampfer behaupten. Neben einer lebhaften Güter- und Personenschiffahrt innerhalb Bihars bildete sich ein Dreiecksverkehr zwischen Bihar, Assam und Calcutta heraus. Jeden Monat gingen einige tausend Tonnen landwirtschaftlicher Güter wie Zucker, Reis und Brotgetreide von Bihar zu den Plantagengebieten Assams. Auf dem Brahmaputra fuhren die Schiffe bis Dibrugarh. ${ }^{1}$ In Assam wurden Holz, Jute und später auch Tee für Calcutta zur Verarbeitung und zum Export geladen. Von Calcutta aus brachten die Schiffe importierte Fertigwaren oder Erzeugnisse der Industrie am Hooghly nach Patna. Sollten die Waren weiter geführt werden, so mußten sie in Patna auf kleinere Heckraddampfer umgeladen werden, da die normale Mindestwassertiefe zwischen Patna und Buxar weniger als $1 \mathrm{~m}$ betrug.

Die Grenzziehung nach der Teilung Indiens und Pakistans brachte es mit sich, daß der Verkehr mit Assam über pakistanisches Gebiet geführt werden mußte. Im Verkehr Bihars mit Calcutta war der Bhagirathi, der auf indischem Gebiet fließt, nur für die Dauer von zwei Monaten im Jahr in der Regenzeit zu benutzen; für den Rest des Jahres ging auch hier der Weg durch Ost-Pakistan. Dies trug entscheidend zum Rückgang der Gangesschiffahrt bei. Darüber hinaus scheinen Bemühungen der Joint Steamer Services um eine Rationalisierung des Betriebes durch Schließung unwirtschaftlicher Stationen und um größere Zuschüsse der Staaten Bihar und Uttar-Pradesh für flußbauliche Arbeiten nicht den gewünschten Erfolg gehabt zu haben. Zuletzt bestand nur noch eine regelmäßige Verbindung zwischen Patna und Calcutta und ein Zubringerdienst auf dem Gogra von Patna bis Barhaj-Bazaar. 1957 war der Verkehr bis auf knapp $4500 \mathrm{t}$ - davon je etwa die Hälfte mit Assam und Calcutta - zusammengeschmolzen. ${ }^{2} 1958$ stellten die Joint Steamer Services ihren Betrieb ein, was - von einigen Fährdiensten abgesehen - das Ende der Dampfschiffahrt auf dem Ganges bedeutete. Country boats werden dagegen noch in großer Zahl für kürzere Entfernungen oder Flußüberquerungen auf Ganges, Gogra, Gandak und Abschnitten der Son-Kanäle be-

1 Seit dem Erdbeben in Assam von 1950 kann die Schiffahrt nur noch bis Neamati, etwa $113 \mathrm{~km}$ unterhalb Dibrugarh, vordringen.

2 National Council of Applied Economic Rescarch (Ed.) : Techno-Economic Survey of Bihar, Vol. 1, New Delhi 1959, S. 65 ff. 
nutzt. Es handelt sich dabei aber um einen Gelegenheitsverkehr ohne festen Fahrplan, der zudem mit großen Risiken behaftet ist, die nicht nur aus den Gefahren des Flusses, sondern auch aus dem Fehlen einer Organisation - Frachtpapiere oder Versicherung gibt es natürlich nicht - resultieren.

Die schnelle Industrialisierung in Teilen Indiens seit der Unabhängigkeit des Landes hat den Transportbedarf erheblich anschwellen lassen und zu einer Überlastung der Eisenbahnen geführt. Von indischen Regierungsstellen ist daher in den letzten Jahren untersucht worden, ob die Wiederaufnahme der Großschiffahrt auf dem Ganges zu einer Entlastung der Bahnen beitragen könnte. Schließlich hat Bihar über $600 \mathrm{~km}$ Wasserwege, die sich ohne allzu großen Aufwand ausbauen ließen, wenn man sich mit flachgehenden Schiffen begnügte. Dazu sind Baggerarbeiten von Oktober bis Mai und Fahrrinnenmarkierungen notwendig. Ferner müßte der Versuch unternommen werden, die Fahrwasserrinne möglichst festzulegen. Auch der Ausbau und Unterhalt der Landeplätze und ihrer landseitigen Zufahrtwege sowie die Errichtung von Lagerschuppen sind erforderlich. Allerdings wäre die in diesem Rahmen vorgesehene Schiffahrt auf den Strecken, wo die Eisenbahn als Konkurrent auftritt, kaum wettbewerbsfähig. Selbst unter günstigen Voraussetzungen wird man nicht den Verkehr großer europäischer Flüsse erwarten dürfen, bei denen der überwiegende Teil der Frachten aus industriellen Massengütern besteht, die im Direktverkehr von Wasseranliegern umgeschlagen werden. Bodenschätze sind in Flußnähe nicht vorhanden; die wenige Industrie des fast ganz auf der Landwirtschaft basierenden Gebietes beschäftigt sich mit der Verarbeitung landwirtschaftlicher Produkte. ${ }^{3}$ Sie liegt nicht am Fluß, weil dessen Ufer entweder steil und trocken, dann jedoch unbefestigt, oder flach, dann aber häufig überschwemmt sind. Die wichtigste Verbindung zu den Bergbaugebieten Chota-Nagpurs für die Zufuhr von Kohle und Kunstdünger kann die Schiffahrt nicht herstellen. Transporte sind demnach überwiegend aus dem landwirtschaftlichen Bereich zu erwarten. Sie würden sich hauptsächlich auf kürzeren Strecken bewegen und eine durch bessere Verkehrsmittel ermöglichte Konsolidierung oder Ausweitung des bestehenden Country boat-Verkehrs bedeuten. Das Potential liegt bei etwa 200000 t pro Jahr. ${ }^{4}$ Transportgüter sind Reis, Getreide, Ölsaaten, Tabak, Jute, $^{5}$ Baumwollgüter und Vieh. ${ }^{6}$ Auch von den zahlreichen in flußangrenzenden Distrikten gelegenen Zuckerfabriken, die sich schon früher der Schiffahrt bedienten, sind größere Gütermengen zu erwarten. Nord-Bihar gehört zu den wichtigsten Zuckerproduzenten der Indischen Union, und während der Erntezeit sind die Eisenbahnen nicht in der Lage, den plötzlich anschwellenden Transportbedarf zu befriedigen. Die geplante Verwendung der Bagasse, eines Nebenproduktes der Zuckergewinnung, zur Papiererzeugung könnte weiteren Verkehr bringen. Wahrscheinlich wird sich die Schiffahrt neben der Eisenbahn in einigem Umfang in die Abfuhr der Produkte der $10 \mathrm{~km}$ vom Ganges entfernt gelegenen Ölraffinerie bei Barauni einschalten können.

3 Für die vereinzelten anderen Industrien, wie die Bata-Schuhfabriken, kommt der Flußtransport ohnehin kaum in Frage.

4 Vgl. : National Council of Applicd Economic Rescarch: ¿Ganga Traffic Survey), New Delhi, 1960.

5 Der Anbau von Jute hat in den Distrikten von Nord-Bihar besonders in Purnea, seit der Teilung des Landes und dem Verlust der ostbengalischen Rohstoffbasis für die Jutefabriken Calcuttas erheblich zugenommen. Der Transport aus diesen neuen Anbaugebieten nach Calcutta liegt ganz bei der Eisenbahn. Die Zufuhr erfolgt teilweise mit country boats zu Bahnstationen auf dem Südufer des Ganges. Wenn die Wasserstraßenverbindung über den Bhagirathi nach Calcutta verbessert würde, wäre mit einer Verlagerung fast aller Jutetransporte auf den Wasserweg zu rechnen.

6 Heute wird das Vieh noch in Herden von 4o bis 5o Stück von Händlern, die es auf den Märkten Nord-Bihars aufgekauft haben, nach Süd-Bihar zum Verkauf getrieben. Der Weg führt über die Pontonbrücken bei Darauli über den Gogra und bei Buxar über den Ganges. 


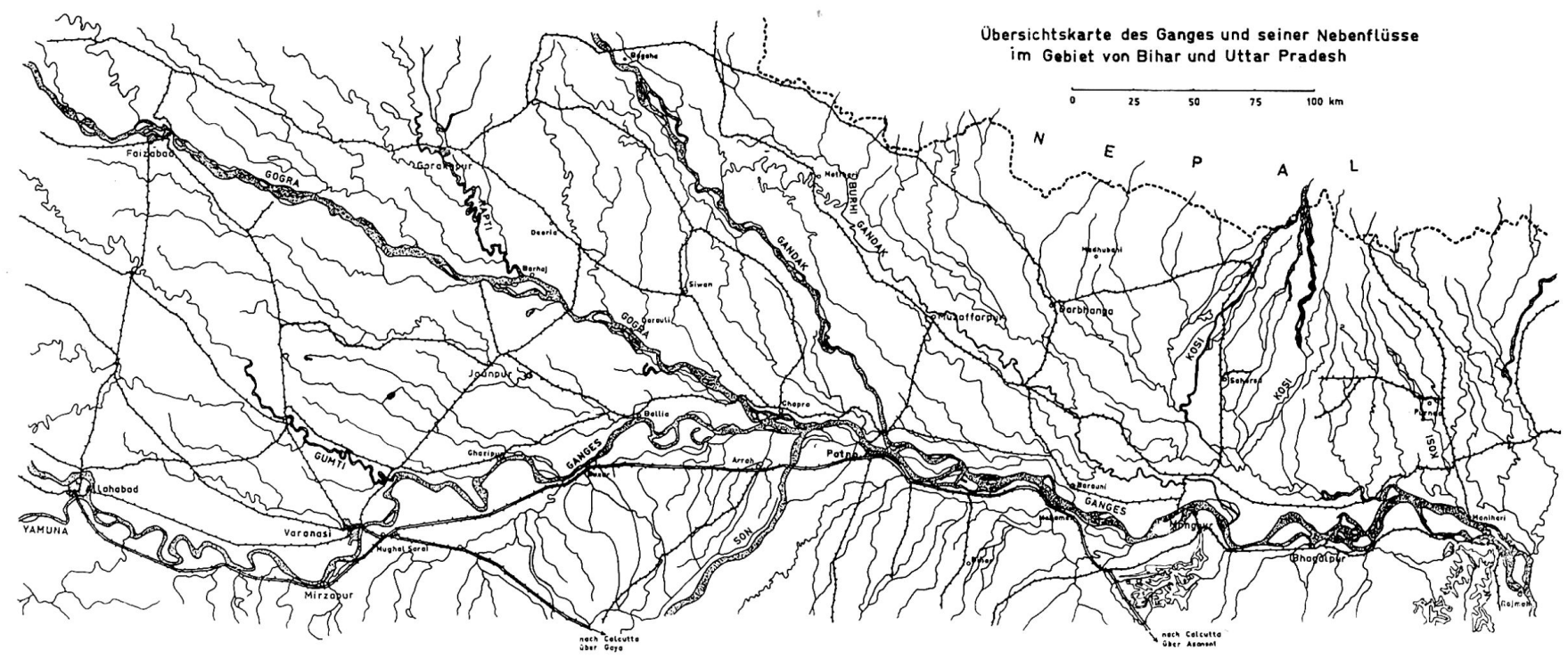


Ferner darf mit Transporten von Steinen aus den Rajmahal-Bergen für Straßenbauten in Nord-Bihar gerechnet werden.

Vor allem für Nord-Bihar wird die Wiederaufnahme der Ganges-Schiffahrt einen erheblichen Vorteil bringen, da dieses Gebiet vom Eisenbahn- und Straßenverkehr nur unzureichend bedient wird. Während der südlich des Flusses gelegene Landesteil günstige Eisenbahnverbindungen besitzt, bestand keine Brücke auf über $900 \mathrm{~km}$ zwischen Benares und Hardinge-Bridge in Ost-Pakistan. Der Eisenbahnverkehr mit Nord-Bihar war auf Fähren angewiesen, was zu langen Wartezeiten führte. ${ }^{7}$ Das Fehlen einer direkten Verbindung mit dem Eisenbahnnetz des übrigen Indien hat zum Bau von eingleisigen Strecken geführt, die mit ihrer Einmeterspur nicht den Hauptstrecken des Landes entsprechen. Die Verkehrsverhältnisse werden noch durch die vom Himalaya kommenden nördlichen Gangeszuflüsse verschlechtert, die beim Eintritt in die Ebene ihr Gefälle verlieren und verwildern. Flußverlegungen und gewaltige Überschwemmungen haben Brückenbauten erschwert und machten es notwendig, die Eisenbahnlinien über weite Strecken auf Dämmen zu bauen. So ist Nord-Bihar verkehrsmäßig eines der am stärksten benachteiligten Gebiete Indiens. Der Bau einer Eisen- und Straßenbrücke bei Mokameh, etwa $100 \mathrm{~km}$ unterhalb Patna, die 1959 fertiggestellt wurde, hat die Verhältnisse erheblich verbessert und die beiden Landesteile einander nähergerückt. Im Bereich eines Brückenkopfes wird diese neue Verbindung der Schifffahrt zwar einige Konkurrenz machen, aber die Benützung der Mokameh-Brücke bedeutet für den Verkehr zwischen großen Teilen Nord- und Süd-Bihars einen derartigen Umweg, daß gerade beim Transport landwirtschaftlicher Güter noch ein ausreichendes Betätigungsfeld für die Flußschiffahrt bleibt. Das gilt besonders dann, wenn die landseitigen Zufahrtswege besser ausgebaut sind und an den Landestellen ein reibungsloser Umschlag gesichert ist.

Eine ganz neue Situation könnte sich schließlich nach dem Bau eines bei Farraka am Ganges an der Grenze zwischen Bihar und Bengalen geplanten Dammes ergeben, der eine ganzjährige Schiffahrt auf indischem Gebiet über den Bhagirathi gestatten soll. Eine solche direkte Verbindung mit Calcutta und dem Industriegebiet am Hooghly würde dem Ganges eine neue Bedeutung als Verkehrsweg und der Schiffahrt einen erheblichen Auftrieb geben.

\section{NAVIGATION ON THE GANGES}

India's rivers have been considered almost only in regard to their importance for irrigation; the economic value they might offer as waterways was quite neglected. Partly due to this fact, the steamer lines which operated on the river Ganges and its northern tributaries Gogra and Gandak for more than a century had to suspend their services a few years ago. Only recently new attention has been focused on these rivers as means for modern transport, due to the pressure on railway communications caused by the rapid industrialization in some parts of the country. Navigation, however, encounters great difficulties which are due to the monsoonal regime with its great difference of water discharge during the dry period and the rainy season of the year and due also to the unstable course of the rivers. The development of river traffic is also handicapped by the fact that Calcutta, the big manufacturing and trading centre, can only be reached via East-Pakistan. Even under these conditions a moderate traffic of mainly agricultural goods could be expected over shorter distances between the riverine districts. This river transport would be of great importance to North Bihar, a region whose development has been hampered by inadequate transport facilities in the past.

7 Selbst im Personenverkehr braucht man fünf Stunden, um die rund $60 \mathrm{~km}$ von Patna bis Muzzafarpur zurückzulegen. 